\title{
Can I talk about your health?
}

\author{
Abeer Arain* \\ Department of Internal Medicine, Carle Foundation Hospital Program, Carle Forum, LL, MC-474, Urbana, Illinois, USA
}

Obesity is a global epidemic, and its prevalence is increasing in every part of the world.1 In 2014, more than 2.1 billion people were overweight or obese, with an economic burden of $\$ 2.0$ trillion or $2.8 \%$ of the gross domestic product (GDP) globally [1]. Obesity is rather like a packaged deal; where we see obesity, we see the concurrent rise in health conditions such as diabetes, hypertension, stroke, osteoarthritis, renal diseases, cancer and so forth. Obesity threatens quality-of-life and causes disabilities. While advances have led to the development of new medications and complex surgical techniques that promote weight loss, it is awareness of healthy eating habits and dietary education that remain the most important factors in helping control body weight.

While nutrition education has been a part of medical school curriculum since 1962 when instituted by the American Medical Association's (AMA) Council on Foods and Nutrition, a 2009 survey reported $62-73 \%$ of US medical schools are not meeting the minimum standards of 25 hours of nutrition instruction as recommended by the National Academy of Sciences (NAS) [2]. Nutrition knowledge appears confined largely to books and exams; according to one study, doctors engage in nutrition counseling with patients only $11 \%$ of the time [3]. In teaching hospitals, where residents work closely with patients, it is crucial the residents develop a comprehensive knowledge of nutrition science and apply that knowledge to clinical practice. Unfortunately, they are under the misconception that nutrition counseling is not their role, but rather the function of dieticians. Inadequate knowledge of nutrition or not feeling competent enough to address nutritional concerns is also common. One study highlights that $67 \%$ of physicians acknowledged lack of training in dietary counseling skills and $62 \%$ claimed deficits in nutrition knowledge [3]. The same study reported that $77 \%$ of residents agree that nutrition assessment should be included in primary care visits [3], yet less than half of physicians say they routinely discuss weight loss plans or provide dietary counseling to their patients.

However, this brings up an interesting point: are physicians themselves (including residents) healthy enough to provide this counseling? Are they, in fact, the models of healthy living their patients believe them to be? It is a well-known fact that physicians' attitudes and personal habits may have a significant impact on their practice of nutrition and lifestyle counseling. If the physicians are consuming a high-calorie diet, eating fast food, avoiding home cooked meals due to

Copyright: (C2018 Arain A. This is an open-access article distributed under the terms of the Creative Commons Attribution License, which permits unrestricted use, distribution, and reproduction in any medium, provided the original author and source are credited. busy schedules and simply unable to differentiate between real food and "food-like substances," can they provide proper nutrition education to their patients?

As health care providers, we encounter patients almost every day who are paying the price of their poor eating habits. Acute conditions are treated in the inpatient setting, where abnormal lab values are fixed, and the patient is discharged with recommended follow-up with primary care. In the outpatient setting the discharge notes are reviewed, medication refills provided, and referrals made. However, in most cases, other than questioning the restricted elements of a diet such as sodium or fluid intake, inquiry of the patient's daily eating habits availability of food, affordability of fresh produce, meal preparation at home versus consuming fast food - is largely overlooked by physicians. This is regretful because, as the literature supports, patients consider clinic physicians to be the credible source of nutrition and desire to discuss their dietary plans with their primary provider [4].

There is an urgent need for nutritional knowledge among young physicians-in-training and a more urgent need for physicians to promote healthy eating habits to their patients. Encouraging healthy eating choices among residents will, in turn, foster the importance of educating patients regarding lifestyle changes. An online nutrition course, such as the one recently launched by the Gaples Institute in partnership with the AMA [5], plus a brief nutrition survey to assess resident nutritional knowledge can serve to help physicians educate patients on the many dangers of obesity.

\section{References}

1. Tremmel M, Gerdtham UG, Nilsson PM, Saha S (2017) Economic burden of obesity: A systemic literature review. Int J Environ Res Public Health 14: 435. [Crossref]

2. Lazarus K, Weinsier RL, Boker JR (1993) Nutrition knowledge and practices of physicians in a family-practice residency program: the effect of an education program provided by a physician nutrition specialist. Am J Clin Nutr 58: 319-325. [Crossref]

3. Vetter ML, Herring SJ, Sood M, Shah NR, Kalet AL (2008) What do residen physicians know about nutrition? An evaluation of attitudes, self-perceived proficiency and knowledge. J Am Coll Nutr 27: 287-298. [Crossref]

4. Schlair S, Hanley K, Gillespie C, Disney L, Kalet A, et al. (2012) How medical students' behaviors and attitudes affect the impact of a brief curriculum on nutrition counseling. J Nutr Edu and Behavior 44: 653-657. [Crossref]

5. Gaples Institute for Integrative Cardiology. A non-profit advocating natural strategies balanced with conventional medicine.

${ }^{*}$ Correspondence to: Abeer Arain, MD, MPH, Resident (PGY3), Department of Internal Medicine, Carle Foundation Hospital Program, Carle Forum, LL, MC474, Urbana, Illinois, USA, E-mail: aarain@illinois.edu

Received: September 21, 2018; Accepted: September 28, 2018; Published: October 01, 2018 